\title{
Affine Projection and Recursive Least Squares Adaptive Filters Employing Partial Updates
}

\author{
Patrick A. Naylor and Andy W.H. Khong \\ Department of Electrical and Electronic Engineering, Imperial College London \\ Email: \{p.naylor, andy.khong\}@imperial.ac.uk
}

\begin{abstract}
We present order $K$ affine projection and recursive least squares adaptive filters employing partial update schemes. The starting point of the work is the MMax tap-selection criterion in which, given a filter length $L$, only $M$ coefficients are updated that correspond to the $M$ largest magnitude elements of the regression vector. We extend this approach from its existing form of MMax-NLMS to new affine projection and recursive least squares schemes with supporting analysis and simulation results. We discuss the computational complexity of these approaches for two alternative sort procedures. Finally, we extend the MMax criterion to a multichannel case by introducing an exclusivity constraint and show the effectiveness of the resulting XM tapselection criterion for application to stereophonic acoustic echo cancellation.
\end{abstract}

\section{INTRODUCTION}

Adaptive filters have been popular in diverse fields such as communications, radar and biomedical engineering. In system identification applications such as shown in Figure 1, these filters employ adaptive algorithms to model the unknown channel. For acoustic applications, these channels correspond to the impulse response of a room which is typically around 100-500 ms for an office such that with a sampling frequency of $8 \mathrm{kHz}$, this corresponds to between 800 and 4000 filter coefficients. Consequently adaptive algorithms such as the normalized least mean squares (NLMS), affine projection (AP) and recursive least squares (RLS) algorithms require high computational complexity for implementation.

Partial update algorithms such as [1][2][3] aim to reduce computational complexity by updating only a subset of filter coefficients in each time iteration. The distribution of the elemental amplitudes in the update vector of, for example, the NLMS algorithm is dependent on the input signal so that, in the case of a speech signal, many of the elements of the update vector are therefore close to zero. This rationale provides the motivation for the MMax tap-selection criterion originally proposed by [4] in which, for a filter of length $L$, only coefficients corresponding to the $M$ largest magnitude elements of the regression vector are updated at each iteration. When MMax tap-selection is applied in adaptive echo cancellation, for example, the resulting MMax-NLMS algorithm can be shown [4] to give the same performance as a fully updated NLMS in terms of final misadjustment but with a reduction in convergence speed. A computational saving of $(L-M)$ tap updates is made at the cost of sorting for the $M$ largest magnitude taps. An efficient approximate sort [5] has been proposed which reduces this cost.

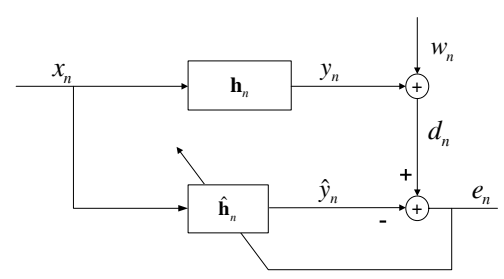

Fig. 1. Single channel system identification employing adaptive filter

Whereas for a single channel case, partial updating schemes are motivated by the reduction in computational complexity, these algorithms have recently been employed successfully in the area of stereophonic acoustic echo cancellation (SAEC) [6] to achieve interchannel decorrelation. It has been shown that for realistic applications, the high interchannel coherence between the two channels results in an ill-conditioned autocorrelation matrix of the tap-input vectors [7]. Consequently, the adaptive filters suffer from slow convergence. To mitigate this problem, a non-linear (NL) preprocessor has been proposed in [8]. The exclusive maximum (XM) tap-selection was proposed [9] in combination with the NL preprocessor such that the XM tap-selection jointly maximizes the MMax criterion whilst minimizing the the interchannel coherence at each iteration. XM tap-selection addresses the minimum coherence condition by constraining tap-selection to be exclusive between the two channels so that the same coefficient index may not be selected in both channels as will be discussed in Section V.

The rest of this paper is organized as follows: In Section II, we extend the MMax tap-selection criterion to the affine projection (MMax-AP) and recursive least squares (MMaxRLS) algorithms. We will discuss the computational complexity associated when these proposed algorithms utilize the SORTLINE [10] and the efficient Short-sort [5] algorithms in Section III. Section IV presents the misalignment analysis for both the MMax-NLMS and MMax-RLS algorithms. Simulation results and conclusion will be presented in Section VI and Section VII respectively.

\section{MMaX AlgORITHMS}

\section{A. MMax-NLMS}

The MMax-NLMS algorithm was originally proposed in [3][4] with the aim of reducing the computational complexity of the NLMS algorithm. With reference to Figure 1, 
we first define measurement noise, filter coefficients and tapinput vector as $w_{n}, \hat{\mathbf{h}}_{n}=\left[\hat{h}_{n}(0) \hat{h}_{n}(1) \ldots \hat{h}_{n}(L-1)\right]^{T}$ and $\mathbf{x}_{n}=\left[x_{n}(0) x_{n}(1) \ldots x_{n}(L-1)\right]^{T}$ respectively. In the MMaxNLMS algorithm, for an adaptive filter of length $L$, only taps corresponding to the $M$ largest magnitude tap-inputs are updated at each iteration such that

$$
\hat{\mathbf{h}}_{n+1}=\hat{\mathbf{h}}_{n}+\mathbf{Q}_{n} \frac{\mu \mathbf{x}_{n} e_{n}}{\left\|\mathbf{x}_{n}\right\|^{2}+\delta}
$$

where $\mathbf{Q}_{n}=\operatorname{diag}\left\{\mathbf{q}_{n}\right\}$ is the tap-selection matrix with elements given by

$$
q_{n}(i)= \begin{cases}1 & \left|x_{n}(i)\right| \in\left\{M \text { maxima of }\left|\mathbf{x}_{n}\right|\right\} \\ 0 & \text { otherwise }\end{cases}
$$

for $i=1,2, \ldots, L$, and the adaptive step-size is $\mu$. The error signal is given by $e_{n}=d_{n}-\hat{\mathbf{h}}_{n}^{T} \mathbf{x}_{n}$.

It has been shown in [6] that the reduction in convergence rate due to tap-selection can be explained by introducing a measure $\mathcal{M}_{n}$, as the ratio of the energy of the $M$ selected tap-inputs to the energy of all the tap-inputs in the tap-input vector such that

$$
\mathcal{M}_{n}=\frac{\left\|\mathbf{Q}_{n} \mathbf{x}_{n}\right\|^{2}}{\left\|\mathbf{x}_{n}\right\|^{2}} .
$$

As a result of a graceful reduction of $\mathcal{M}_{n}$ for $0.5 L \leq M \leq L$, the MMax-NLMS algorithm only suffers a small reduction in the rate of convergence for this range of $M$.

\section{B. MMax-Affine Projection Algorithms}

The affine projection algorithm [11] incorporates multiple projections by concatenating past input vectors from time iteration $n$ to time iteration $n-K+1$ where $K$ is defined as the projection order. We first define the subselected tap-input vector $\widetilde{\mathbf{x}}_{n}=\mathbf{Q}_{n} \mathbf{x}_{n}$. The subselected and full tap-input matrix are then denoted respectively as

$$
\begin{aligned}
\widetilde{\mathbf{X}}_{n} & =\left[\begin{array}{llll}
\widetilde{\mathbf{x}}_{n} & \widetilde{\mathbf{x}}_{n-1} & \ldots & \widetilde{\mathbf{x}}_{n-K+1}
\end{array}\right]^{T} \\
\mathbf{X}_{n} & =\left[\begin{array}{llll}
\mathbf{x}_{n} & \mathbf{x}_{n-1} & \ldots & \mathbf{x}_{n-K+1}
\end{array}\right]^{T} .
\end{aligned}
$$

Using (4), (5) and following the same approach as [11], the tap update equation for the MMax-AP algorithm is given as

$$
\hat{\mathbf{h}}_{n+1}=\hat{\mathbf{h}}_{n}+\mu \tilde{\mathbf{X}}_{n}^{T}\left[\mathbf{X}_{n} \mathbf{X}_{n}^{T}+\delta \mathbf{I}\right]^{-1} \mathbf{e}_{n}
$$

where $\mathbf{e}_{n}=\mathbf{d}_{n}-\mathbf{X}_{n} \hat{\mathbf{h}}_{n}, \mathbf{d}_{n}=\left[\begin{array}{lll}d_{n} & d_{n-1} \ldots & d_{n-K+1}\end{array}\right]^{T}$ and $\mathbf{I}$ is a $K \times K$ identity matrix. It can be seen from (6) that for projection order $K=1$, MMax-AP is equivalent to MMax-NLMS.

Even though the matrix $\widetilde{\mathbf{X}}_{n}$ is formed by subselecting the tap-input vector, MMax-AP in general cannot be classified as a partial-update algorithm. This is because the length $L$ column vector $\widetilde{\mathbf{X}}_{n}^{T}\left[\mathbf{X}_{n} \mathbf{X}_{n}^{T}\right]^{-1} \mathbf{e}_{n}$ is fully populated and therefore every coefficient in $\hat{\mathbf{h}}_{n}$ will be updated at each iteration.

\section{MMax-RLS Algorithm}

The tap-update equation for RLS is given as

$$
\hat{\mathbf{h}}_{n+1}=\hat{\mathbf{h}}_{n}+\mathbf{k}_{n} e_{n}
$$

where the Kalman gain $\mathbf{k}_{n}$ is

$$
\mathbf{k}_{n}=\frac{\lambda^{-1} \mathbf{R}_{n-1}^{-1} \mathbf{x}_{n}}{1+\lambda^{-1} \mathbf{x}_{n}^{T} \mathbf{R}_{n-1}^{-1} \mathbf{x}_{n}}
$$

such that $0 \ll \lambda<1$ is defined as the forgetting factor and the time-averaged autocorrelation matrix is defined by

$$
\mathbf{R}_{n}=\sum_{i=1}^{n} \lambda^{n-i} \mathbf{x}_{i} \mathbf{x}_{i}^{T} .
$$

As we shall show in Section VI, direct extension of the MMax tap-selection approach achieved by sorting the magnitude of $\mathbf{k}_{n}$ in (7) will not achieve the desired convergence for non-stationary signals such as speech. This is because the Kalman gain depends on previous values of the time-averaged inverse correlation matrix $\mathbf{R}^{-1}$ as shown in (8) such that the statistical nature of non-stationary signals is not preserved from one iteration to the next. For stationary signals however, the statistics remain consistent throughout time iterations and hence convergence may be achieved. For speech signals, our approach will be to form $\widetilde{\mathbf{R}}_{n}$ using the subselected tap-input vector $\widetilde{\mathbf{x}}_{n}=\mathbf{Q}_{n} \mathbf{x}_{n}$. This ensures that the subsampled input vectors propagate consistently through the memory of the algorithm. To derive the MMax-RLS algorithm, we rewrite (9) in terms of the subselected tap-input vector recursively as

$$
\begin{aligned}
\widetilde{\mathbf{R}}_{n} & =\widetilde{\mathbf{X}}_{n} \Lambda_{n} \widetilde{\mathbf{X}}_{n}^{T} \\
& =\lambda \widetilde{\mathbf{R}}_{n-1}+\widetilde{\mathbf{x}}_{n} \widetilde{\mathbf{x}}_{n}^{T}
\end{aligned}
$$

where $\widetilde{\mathbf{X}}_{n}=\left[\begin{array}{lll}\widetilde{\mathbf{x}}_{1} & \widetilde{\mathbf{x}}_{2} \ldots \widetilde{\mathbf{x}}_{n}\end{array}\right]^{T}$ and $\Lambda=\operatorname{diag}\left[\begin{array}{ll}\lambda^{n} & \lambda^{n-1} \ldots \lambda\end{array}\right]$. The cross-correlation vector may be expressed recursively as

$$
\begin{aligned}
\widetilde{\Theta}_{n} & =\widetilde{\mathbf{X}}_{n} \Lambda_{n} \mathbf{d}_{n} \\
& =\lambda \widetilde{\Theta}_{n-1}+\widetilde{\mathbf{x}}_{n} d_{n}
\end{aligned}
$$

such that $\mathbf{d}_{n}=\left[\begin{array}{lll}d_{1} & d_{2} \ldots d_{n}\end{array}\right]^{T}$. Using the matrix inversion lemma and following the approach of [11] gives

$$
\widetilde{\mathbf{R}}_{n}^{-1}=\frac{1}{\lambda}\left[\widetilde{\mathbf{R}}_{n-1}^{-1}-\widetilde{\mathbf{k}}_{n} \widetilde{\mathbf{x}}_{n}^{T} \widetilde{\mathbf{R}}_{n-1}^{-1}\right]
$$

where the modified Kalman gain is given by

$$
\widetilde{\mathbf{k}}_{n}=\frac{\lambda^{-1} \widetilde{\mathbf{R}}_{n-1}^{-1} \widetilde{\mathbf{x}}_{n}}{1+\lambda^{-1} \widetilde{\mathbf{x}}_{n}^{T} \widetilde{\mathbf{R}}_{n-1}^{-1} \widetilde{\mathbf{x}}_{n}} .
$$

The MMax-RLS tap-update equation is then given by

$$
\hat{\mathbf{h}}_{n+1}=\hat{\mathbf{h}}_{n}+\widetilde{\mathbf{k}}_{n} e_{n} .
$$

Similar to the XMNL-AP, the XMNL-RLS algorithm in general updates all the taps at each iteration since the Kalman gain vector $\widetilde{\mathbf{k}}_{n}$ is a fully populated column vector except in special cases where there exist any null rows $\mathbf{R}_{n}^{-1}$.

\section{Computational CompleXity}

The MMax tap-selection procedure selects the $M$ largest tap-inputs at each time iteration. This sorting operation can be achieved efficiently using for example the SORTLINE [10] or the Short-sort [5] routine. The Short-sort tap-selection routine operates by considering two regions of the impulse response, one of length $S$ and one of length $L-S$, such that all taps are updated at each iteration in the first region. In the second region, only $A$ out of $L-S$ taps are updated. The resultant SM-NLMS algorithm [5] selects $A$ out of $S$ taps in the first region corresponding to the $A$ largest samples in $\left[x_{n}, x_{n-1}, \ldots, x(n-S+1)\right]^{T}$ and then tracks these $A$ largest samples as they propagate through the memory of the filter. Thus the worst-case comparison load using Short-sort 
is $[1+S-A] A / S$ comparisons per iteration compared to $2+2 \log _{2} L$ used in the SORTLINE procedure.

In this Section, we examine the computational complexity of the SM-NLMS, MMax-NLMS, MMax-AP and MMaxRLS algorithms. We define complexity as the total number of multiplications and comparisons per sample period. The computational complexity for each algorithm is summarized as shown below:

$$
\begin{aligned}
& \text { MMax - NLMS (Short - sort) }: L+S+\frac{A}{S}(L+1-A) \\
& \text { MMax - NLMS (SORTLINE) }: L+M+3+2 \log _{2} L \\
& \text { MMax - AP (SORTLINE): }(M+L) K+7 K^{2}+2 \\
&+2 \log _{2} L \\
& \text { MMax - RLS (SORTLINE) }: L(L+3 M+2)+M+3 \\
&+2 \log _{2} L .
\end{aligned}
$$

\section{MisalignMENT ANALYSis}

In this analysis, we consider algorithms of the form

$$
\hat{\mathbf{h}}_{n+1}=\hat{\mathbf{h}}_{n}+\Gamma_{n} \mathbf{x}_{n} e_{n}
$$

where $\Gamma_{n}$ is an $L \times L$ adaptation control matrix. For NLMS, $\Gamma_{n}=\mu_{n} \mathbf{I}=\frac{\mu}{\mathbf{x}_{n}^{T} \mathbf{x}_{n}} \mathbf{I}$ while for RLS, the Kalman gain vector may be expressed as $\mathbf{k}_{n}=\mathbf{R}_{n}^{-1} \mathbf{x}_{n}$ [11] and thus $\Gamma_{n}=\mathbf{R}_{n}^{-1}$ where $\mathbf{R}_{n}$ is as defined in (9). For the purpose of analysis, we assume that the dimension of $\hat{\mathbf{h}}_{n}$ is chosen to match the dimension of a time-invariant system $\mathbf{h}_{n}$. We define the misalignment vector $\mathbf{v}_{n}=\hat{\mathbf{h}}_{n}-\mathbf{h}_{n}$ and hence the error signal $e_{n}=w_{n}-\mathbf{x}_{n}^{T} \mathbf{v}_{n}$ where $w_{n}$ is measurement noise. Thus it follows that $\mathbf{v}_{n+1}$ can be expressed as

$$
\mathbf{v}_{n+1}=\left[\mathbf{I}-\Gamma_{n} \mathbf{x}_{n} \mathbf{x}_{n}^{T}\right] \mathbf{v}_{n}+\Gamma_{n} \mathbf{x}_{n} w_{n}
$$

and defining $\mathbf{R}_{\mathbf{v}, n+1}=E\left[\mathbf{v}_{n+1} \mathbf{v}_{n+1}^{T}\right]$,

$$
\begin{aligned}
\mathbf{R}_{\mathbf{v}, n+1}= & \mathbf{R}_{\mathbf{v}, n}-E\left[\Gamma_{n} \mathbf{x}_{n} \mathbf{x}_{n}^{T}\right] \mathbf{R}_{\mathbf{v}, n}-\mathbf{R}_{\mathbf{v}, n} E\left[\mathbf{x}_{n} \mathbf{x}_{n}^{T} \Gamma_{n}^{T}\right] \\
& +E\left[\Gamma_{n} \mathbf{x}_{n} \mathbf{x}_{n}^{T} \mathbf{v}_{n} \mathbf{v}_{n}^{T} \mathbf{x}_{n} \mathbf{x}_{n}^{T} \Gamma_{n}^{T}\right] \\
& +E\left[\Gamma_{n} \mathbf{x}_{n} \mathbf{x}_{n}^{T} \Gamma_{n}^{T}\right] \sigma_{w}^{2}
\end{aligned}
$$

where $E[$.$] is the expectation operator and E\left[w_{n}\right]=0$.

\section{A. MMax-NLMS Analysis}

The MMax-NLMS employs tap-selection such that $\Gamma_{n}=$ $\mu_{n} \mathbf{Q}_{n}$ where $\mathbf{Q}_{n}$ is a diagonal matrix whose elements are defined in (2). Convergence in the mean for MMax-NLMS can be derived using a contraction mapping approach [12]: $0<\left\|E\left[\mathbf{I}-\mu_{n} \mathbf{Q}_{n} \mathbf{x}_{n} \mathbf{x}_{n}^{T}\right]\right\|<1$ which implies for i.i.d. $x_{n}$

$$
0<\mu_{n}<\frac{2}{\sum_{i=0}^{L-1} q_{n}(i) x_{n-i}^{2}}
$$

For convergence in the mean square, we start by considering (21) and we note that $q_{n}(i)$ are not independent of $\mathbf{x}_{n}$ as they ensure that only the $M$ largest elements in $\left|\mathbf{x}_{n}\right|$ are selected. The selected samples are assumed to have zero mean and variance denoted $\kappa$. Assuming that $\mathbf{x}_{n}$ is a white Gaussian sequence such that $\mathbf{x}_{n} \mathbf{x}_{n}^{T}$ is diagonal and using $E\left[\mu_{n}\right]=\frac{\mu}{L \sigma_{x}^{2}}$, we can evaluate the following expressions

$$
\begin{gathered}
E\left[\Gamma_{n} \mathbf{x}_{n} \mathbf{x}_{n}^{T}\right]=E\left[\mathbf{x}_{n} \mathbf{x}_{n}^{T} \Gamma_{n}^{T}\right]=E\left[\mu_{n}\right] E\left[\mathbf{Q}_{n} \mathbf{x}_{n} \mathbf{x}_{n}^{T}\right] \\
=\frac{M}{L} \frac{\mu}{L \sigma_{x}^{2}} \kappa \mathbf{I} \\
\operatorname{tr}\left\{E\left[\Gamma_{n} \mathbf{x}_{n} \mathbf{x}_{n}^{T} \mathbf{v}_{n} \mathbf{v}_{n}^{T} \mathbf{x}_{n} \mathbf{x}_{n}^{T} \Gamma_{n}^{T}\right]\right\} \\
=\frac{\mu^{2}}{L^{2} \sigma_{x}^{4}} \operatorname{tr}\left\{\mathbf{R}_{\mathbf{v}, n}\right\}(L+2) \kappa \sigma_{x}^{2} \\
\operatorname{tr}\left\{E\left[\Gamma_{n} \mathbf{x}_{n} \mathbf{x}_{n}^{T} \Gamma_{n}^{T}\right]\right\}=\frac{M}{L} \frac{\mu^{2}}{L^{2} \sigma_{x}^{4}} \kappa L .
\end{gathered}
$$

Substituting (23)-(25) into (21), we obtain

$$
\begin{aligned}
\operatorname{tr}\left\{\mathbf{R}_{\mathbf{v}, n+1}\right\}= & \operatorname{tr}\left\{\mathbf{R}_{\mathbf{v}, n}\right\}\left\{1-\frac{4 \mu M \kappa}{L^{2} \sigma_{x}^{2}}+(L+2) \frac{4 \mu^{2} M \kappa}{L^{3} \sigma_{x}^{2}}\right\} \\
& +\frac{4 \mu^{2} M}{L^{2} \sigma_{x}^{4}} \kappa \sigma_{w}^{2} .
\end{aligned}
$$

Assuming that $\mathbf{R}_{\mathbf{v}, n}$ is fluctuating around its mean, we define $\mathbf{R}_{\mathbf{v}}$ as the approximately time-invariant autocorrelation matrix of the mean weight error vector $\eta$ thus giving

$$
\eta=\operatorname{tr}\left\{\mathbf{R}_{\mathbf{v}}\right\}=\frac{\mu \sigma_{w}^{2}}{\sigma_{x}^{2} \phi}
$$

where $\phi=1-\mu\left(\frac{2}{L}+1\right)$. It can be seen from (27) that the mean weight error vector is inversely proportional to the signal-tonoise ratio (SNR). Additionally, it can be deduced that the NLMS algorithm is robust to MMax-tap selection such that the same final misalignment can be achieved independently of $M$.

\section{B. MMax-RLS Analysis}

The tap update equation for MMax-RLS may be written as

$$
\hat{\mathbf{h}}_{n+1}=\hat{\mathbf{h}}_{n}+\widetilde{\mathbf{R}}_{n}^{-1} \mathbf{Q}_{n} \mathbf{x}_{n} e_{n} .
$$

In this case, $\Gamma_{n}=\widetilde{\mathbf{R}}_{n}^{-1} \mathbf{Q}_{n}$ in (19) and $\widetilde{\mathbf{R}}_{n}=$ $\sum_{i=1}^{n} \lambda^{n-i}\left[\mathbf{Q}_{i} \mathbf{x}_{i} \mathbf{x}_{i}^{T} \mathbf{Q}_{i}^{T}\right]$. In the limit $n \rightarrow \infty$ and using $\mathbf{Q}_{n} \mathbf{x}_{n} \mathbf{x}_{n}^{T} \mathbf{Q}_{n}^{T}=M \kappa \mathbf{I} / L$, it follows that

$$
E\left[\lim _{n \rightarrow \infty} \widetilde{\mathbf{R}}_{n}\right]=\frac{1}{1-\lambda} \frac{M}{L} \kappa \mathbf{I}
$$

and hence $\Gamma_{n}=(1-\lambda) \frac{L}{M \kappa} \mathbf{Q}_{n}$ giving rise to the following

$$
\begin{aligned}
E\left[\Gamma_{n} \mathbf{x}_{n} \mathbf{x}_{n}^{T}\right]=E\left[\mathbf{x}_{n} \mathbf{x}_{n}^{T} \Gamma_{n}^{T}\right] & =(1-\lambda) \frac{L}{M \kappa} E\left[\mathbf{Q}_{n} \mathbf{x}_{n} \mathbf{x}_{n}^{T}\right] \\
& =(1-\lambda) \mathbf{I} \\
E\left[\Gamma_{n} \mathbf{x}_{n} \mathbf{x}_{n}^{T} \mathbf{v}_{n} \mathbf{v}_{n}^{T} \mathbf{x}_{n} \mathbf{x}_{n}^{T} \Gamma_{n}^{T}\right] & =\frac{(1-\lambda)^{2} L(L+2) \sigma_{x}^{2}}{M \kappa} \mathbf{R}_{\mathbf{v}, n} \\
E\left[\Gamma_{n} \mathbf{x}_{n} \mathbf{x}_{n}^{T} \Gamma_{n}^{T}\right] & =\left[\frac{(1-\lambda) L}{M \kappa}\right]^{2} E\left[\mathbf{Q}_{n} \mathbf{x}_{n} \mathbf{x}_{n}^{T} \mathbf{Q}_{n}\right] \\
& =\frac{(1-\lambda)^{2} L}{M \kappa} \mathbf{I} .
\end{aligned}
$$

Substituting (30)-(32) into (21), we obtain

$$
\begin{aligned}
\mathbf{R}_{\mathbf{v}, n+1}= & \mathbf{R}_{\mathbf{v}, n}-2(1-\lambda) \mathbf{R}_{\mathbf{v}, n}+\frac{(1-\lambda)^{2} L}{M \kappa} \sigma_{w}^{2} \mathbf{I} \\
& +\frac{(1-\lambda)^{2} L(L+2) \sigma_{x}^{2}}{M \kappa} \mathbf{R}_{\mathbf{v}, n}
\end{aligned}
$$




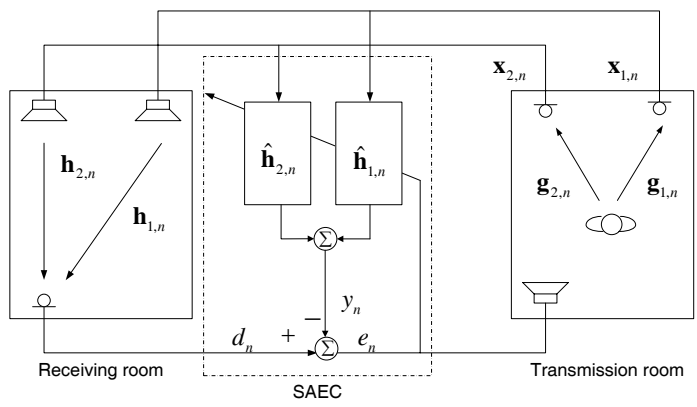

Fig. 2. Schematic diagram of SAEC structure after [8]

As before, we can assume that $\mathbf{R}_{\mathbf{v}, n}$ is fluctuating around its mean and defining $\eta=\operatorname{tr}\left\{\mathbf{R}_{\mathbf{v}}\right\}$ we may then express (33) as

$$
\begin{aligned}
\eta & =\eta-2(1-\lambda) \eta+\frac{(1-\lambda)^{2} L(L+2) \sigma_{x}^{2}}{M \kappa} \eta+\frac{(1-\lambda)^{2} L^{2} \sigma_{w}^{2}}{M \kappa} \\
& =\frac{(1-\lambda) L \sigma_{w}^{2}}{2 \sigma_{x}^{2}\left[\frac{M \kappa}{L \sigma_{x}^{2}}-(1-\lambda)\left(1+\frac{L}{2}\right)\right]} .
\end{aligned}
$$

We can deduce from (34) that, similar to MMax-NLMS, the mean weight error vector for MMax-RLS is inversely proportional to the SNR of the signal. As we shall see in Section VI, the final misalignment of the MMax-RLS is dependent on $M$ such that for fewer tap-selection, the mean weight error vector increases.

\section{Multi-Channel Extension Employing an EXCLUSIVITY CONSTRAINT}

We illustrate an application of the schemes using the example of stereophonic acoustic echo cancellation (SAEC) as shown in Figure 2. Standard adaptive filtering algorithms perform poorly because of the non-uniqueness problem caused by the high inter-channel coherence of the two channel input vectors $\mathbf{x}_{1, n}$ and $\mathbf{x}_{2, n}$ [8]. We first note that direct application of MMax tap-selection will not serve to decorrelate the two tap-input vectors $\mathbf{x}_{1, n}$ and $\mathbf{x}_{2, n}$ because, since they are highly correlated, nearly identical tap-indices will be selected in both filters. We thus propose the use of an extended version of our tap-selection schemes to jointly maximize the MMax criterion whilst minimizing inter-channel coherence by imposing an exclusivity constraint [6]. The resulting exclusive maximum (XM) tap-selection algorithm employs $M=0.5 L$ and achieves the reduction in interchannel coherence by selecting exclusive tap-inputs such that, at each iteration, selection of the same tap index in both channels is not permitted.

Although an exhaustive search of all exclusive tap-selections could be used to find the selection set which maximizes $\mathcal{M}$, a more efficient method can be found by considering $\mathbf{p}_{n}=$ $\left|\mathbf{x}_{1, n}\right|-\left|\mathbf{x}_{2, n}\right|$. The tap-selection with maximum $\mathcal{M}_{n}$ can then be found efficiently by sorting $\mathbf{p}_{n}$.

Consider as a simple example an SAEC system with channels $k=1,2$, adaptive filters each of length $L=4$ and tapinput vectors $\mathbf{x}_{k, n}=\left[x_{k, n}(1) x_{k, n}(2) x_{k, n}(3) x_{k, n}(4)\right]^{T}$. Also consider the example case $p(3)>p(2)>p(1)>p(4)$, for a

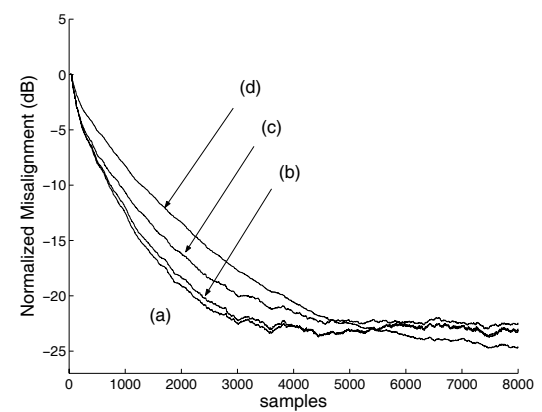

Fig. 3. Normalized misalignment for single channel MMax-AP with $K=2$ and (a) $M=L$, (b) $M=L / 2$, (c) $M=L / 4$ and (d) NLMS

particular $n$th time instant. Since $p(3)+p(2)>p(1)+p(4)$, it follows that

$$
\begin{aligned}
\left|x_{1}(3)\right|+\left|x_{1}(2)\right|+\left|x_{2}(1)\right|+\left|x_{2}(4)\right| & >\left|x_{1}(1)\right|+\left|x_{1}(4)\right| \\
& +\left|x_{2}(2)\right|+\left|x_{2}(3)\right| .
\end{aligned}
$$

Thus at this particular time iteration, selection of taps corresponding to $x_{1}(3), x_{1}(2), x_{2}(1)$ and $x_{2}(4)$ maximizes $\mathcal{M}$ with the minimum coherence constraint satisfied by the exclusivity. In this two channel case, $\mathcal{M}_{n}$ is then defined as $\mathcal{M}_{n}=\frac{\left\|\mathbf{Q}_{n} \mathbf{x}_{n}\right\|^{2}}{\left\|\mathbf{x}_{n}\right\|^{2}}$ where $\mathbf{Q}_{n}=\operatorname{diag}\left\{\left[\mathbf{q}_{1, n}^{T} \quad \mathbf{q}_{2, n}^{T}\right]\right\}$ and $\mathbf{x}_{n}=\left[\begin{array}{ll}\mathbf{x}_{1, n}^{T} & \mathbf{x}_{2, n}^{T}\end{array}\right]^{T}$ such that at each iteration $n$, element $u$ of $\mathbf{q}_{1, n}$ and element $v$ of $\mathbf{q}_{2, n}$ are defined for $u, v=1,2, \ldots, L$ where

$$
\begin{aligned}
& q_{1, n}(u)= \begin{cases}1 & p_{n}(u) \in\{M \text { maxima of } \mathbf{p}\} \\
0 & \text { otherwise }\end{cases} \\
& q_{2, n}(v)= \begin{cases}1 & p_{n}(v) \in\{M \text { minima of } \mathbf{p}\} \\
0 & \text { otherwise. }\end{cases}
\end{aligned}
$$

We propose a method for SAEC employing the XM tapselection in combination with the NL preprocessor so as to improve the convergence rate of algorithms employing the NL preprocessor alone [8]. We shall show in the next Section that the resulting XMNL-based algorithms can give faster rate of convergence.

\section{Simulation Results}

We present simulation results for both single channel and stereophonic AEC applications. In all our simulations, impulse response were generated using the method of images [13] such that the length of the transmission and receiving room were both $G=800$. Adaptive filters were of length $L=256$ and we define normalized misalignment as

$$
\xi_{n}=\left\|\mathbf{h}-\hat{\mathbf{h}}_{n}\right\|^{2} /\|\mathbf{h}\|^{2}
$$

calculated over the first 256 filter coefficients. In all our simulations, independent noise is added to the desired signal such that SNR of $25 \mathrm{~dB}$ is achieved.

\section{A. Single channel AEC}

Figure 3 shows initial convergence in terms of normalized misalignment for single channel MMax-AP of second order $K=2$ with $\mu=0.2$ for (a) $M=L=256$, (b) $M=128$, (c) $M=64$ and (d) NLMS. It can be seen that for $M=$ 


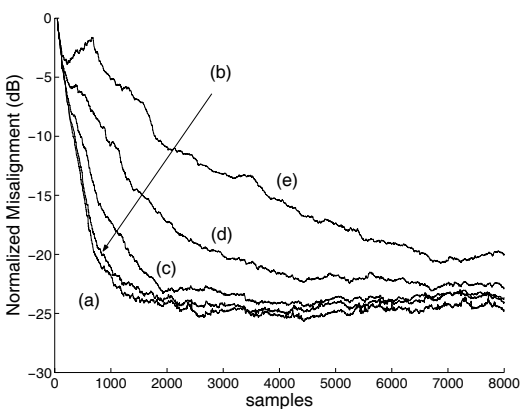

Fig. 4. Normalized misalignment for single channel MMax-RLS (a) $M=L$, (b) $M=L / 2$, (c) $M=L / 4$ and (d) $M=L / 8$ (e) $M=L / 16$

$L$, the misalignment for MMax-AP corresponds to the fullupdate AP as expected. For reducing $M$, the performance of MMax-AP degrades gracefully compared to fully updated AP. Figure 4 shows initial convergence in terms of normalized misalignment for single channel MMax-RLS with (a) $M=$ $L=256$, (b) $M=128$, (c) $M=64$ and (d) $M=32$ and (e) $M=16$ and $\lambda=0.999$. The MMax-RLS algorithm is implemented using (12)-(14). For $M=L / 2$ and $M=L / 4$, the performance of MMax-RLS is close to that of the RLS algorithm. In both MMax-AP and MMax-RLS simulations, white Gaussian noise (WGN) input sequence were used.

\section{B. Stereophonic AEC}

Figure 5 shows an example result of stereophonic AEC in which we incorporated the non-linear preprocessor [14] to both the XM-RLS (XMNL-RLS) and RLS algorithms (NL-RLS). For clarity, the misalignment of only one channel is plotted. The speech input is shown in Figure 5(a). The forgetting factor was $\lambda=0.9996$ while non-linearity constant for NL preprocessor was $\alpha=0.5$. As shown in Figure 5, there is a significant improvement in normalized misalignment of 3 to $6 \mathrm{~dB}$ for the XMNL-RLS compared to that of NL-RLS. This is due to the additional decorrelation property of the XM preprocessor. The performance of XMNL-NLMS has come close to that of the NL-RLS. As well as showing improved performance, the XMNL schemes benefit additionally from the reduced complexity of the partial updating, particularly when the short-sort technique is employed.

\section{CONCLUSION}

In this paper, we have extended the MMax tap-selection criterion to the AP and RLS algorithms. We note that direct extension of the MMax tap-selection approach achieved by sorting the magnitude of Kalman gain in RLS will not achieve the desired convergence for speech input signals. The computational complexity for MMax-AP and MMaxRLS have been discussed. Misalignment analysis for MMaxNLMS and MMax-RLS for the single channel case have been presented. The exclusive maximum (XM) tap-selection was then proposed and applied to SAEC which jointly maximizes the MMax criterion whilst minimizing the interchannel coherence. The resultant XMNL-RLS algorithm achieves faster

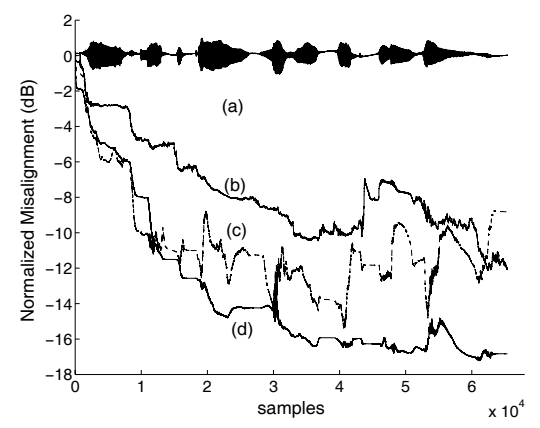

Fig. 5. (a) Speech Signal and normalized misalignment for (b) XMNLNLMS, (c) NL-RLS and (d) XMNL-RLS

convergence of approximately 3 to $6 \mathrm{~dB}$ compared to the use of the NL-preprocessor alone in our simulation example using speech.

\section{REFERENCES}

[1] S. C. Douglas, "Adaptive filters employing partial updates," IEEE Trans. Circuits Syst. II, vol. 44, no. 3, pp. 209-216, Mar. 1997.

[2] _ "Analysis and implementation of the MAX-NLMS adaptive filter," in Proc. Twenty-Ninth Asilomar Conference on Signals, Systems and Computers, vol. 1, 1995, pp. 659-663.

[3] T. Aboulnasr and K. Mayyas, "Complexity reduction of the NLMS algorithm via selective coefficient update," IEEE Trans. Signal Processing, vol. 47, no. 5, pp. 1421-1424, 1999.

[4] _ - "Selective coefficient update of gradient-based adaptive algorithms," in Proc. IEEE Int. Conf. Acoustics Speech Signal Processing, vol. 3, 1997, pp. 1929-1932.

[5] P. A. Naylor and W. Sherliker, "A short-sort M-max NLMS partial update adaptive filter with applications to echo cancellation," in Proc. IEEE Int. Conf. Acoustics Speech Signal Processing, vol. 5, 2003, pp. 373-376.

[6] A. W. H. Khong and P. A. Naylor, "Selective-tap adaptive algorithms in the solution of the non-uniqueness problem for stereophonic acoustic echo cancellation," accepted for publication in IEEE Signal Processing Letters, 2004.

[7] P. Eneroth, S. L. Gay, T. Gansler, and J. Benesty, "A real-time implementation of a stereophonic acoustic echo canceller," IEEE Trans. Speech Audio Processing, vol. 9, no. 5, pp. 513-523, Jul. 2001.

[8] J. Benesty, D. R. Morgan, and M. M. Sondhi, "A better understanding and an improved solution to the specific problems of stereophonic acoustic echo cancellation," IEEE Trans. Speech Audio Processing, vol. 6, no. 2, pp. 156-165, Mar. 1998.

[9] A. W. H. Khong and P. A. Naylor, "Reducing inter-channel coherence in stereophonic acoustic echo cancellation using partial update adaptive filters," in Proc. European Signal Processing Conference, 2004, pp. 405 408.

[10] I. Pitas, "Fast algorithms for running ordering and max/min calculation," IEEE Trans. on Circuits and Systems, vol. 36, no. 6, pp. 795-804, Jun. 1989.

[11] S. Haykin, Adaptive Filter Theory, 4th ed., ser. Information and System Science. Prentice Hall, 2002.

[12] M. Rupp, "Contraction mapping: an important property in adaptive filters," in Proc. Sixth IEEE Digital Signal Processing Workshop, 1994, pp. 273-276.

[13] J. B. Allen and D. A. Berkley, "Image method for efficiently simulating small-room acoustics," J. Acoust. Soc. Amer., vol. 65, no. 4, pp. 943-950, Apr. 1979.

[14] J. Benesty, T. Gansler, D. R. Morgan, M. M. Sondhi, and S. L. Gay, Advances in Network and Acoustic Echo Cancellation. Springer, 2001. 